\title{
Progress of Bibliotherapy Theory and Practice in China
}

\author{
Su Liting ${ }^{1}$, Huang Xiaoli ${ }^{2}$ \\ ${ }^{1}$ School of Management of Hebei Unitded University, Tangshan 063000, China \\ ${ }^{2}$ Library of Hebei Unitded University, Tangshan 063000, China \\ Email:hxlee@vip.163.com
}

\begin{abstract}
As an ancient mental therapy measure, bibliotherapy has been recognized worldwide gradually. In China it has been studied systematically for over twenty years. In this paper, the study situation of bibliotherapy in China was summarized from the aspects of theory and practice, and some suggestions were put forward.
\end{abstract}

Keywords- bibliotherapy; study progress; review

\section{INTRODUCTION}

Reading, not only can be entertained, educational, but also can relieve negative emotions and cure mental illness, which was called "reading therapy", namely a method of making body and mind to obtain relaxation or encouragement, then access to the status of peace and health by reading some books, which can dispel negative emotions [1]. Reading therapy has a history of several hundred years in foreign countries and has been adopted by doctors, consultants, librarians, teachers, parents and so on. The research institutions are mature, applicable people are wide, research objects are diversified. Though reading therapy history in China can go back to ancient times, but the time of study systematically is only over twenty years. Many aspects are still in the exploring stage. In modern society, people live a stressful and fast-paced life, psychological health has aroused the attention of people around the world, the study of reading therapy is also particularly important. So the paper generalized the study history of reading therapy in our country from two aspects of theory and practice for over twenty years, and put forward related suggestions, hoping to provide references for reading therapy research in China.

\section{RESEARCH STATUS}

\section{A. Theory Introduction Stage}

The reading therapy research in China has undergone introduction, exploration, and development. Reports of reading therapy appeared until the 1980s, "reading" belongs to the category of "working entertainment therapy"can be seen originally from Chinese Medical Encyclopedia of Psychiatry under Xia Zhenyi general editorship in 1982 [2]. In the early 1990s, Chinese scholars began to compile and introduce reading therapy to China. The earliest introduction of the concept of reading therapy was Chen Xinchun in his paper Literature Therapy — One of The Contents of The reader Service Work published in the Library Journal in 1991[3]. The paper book, can cure disease marked the beginning systematic study of reading therapy in China published in 1994 by Professor Shen Guchao, known as the "father of reading therapy in China". The reading therapy in the United States was introduced by Yang Bing and Qiu Yousheng through the doctoral research in recent years in the United States [4].In the same year, LingShan, Hua Weina published a paper on reading therapy in medical journal, which recommended reading therapy to the psychiatric field as a adjuvant therapy for emotional disorder for the first time [5].

\section{B. Local Research Stage}

Reading therapy research transformed from the compilation to the localization of theoretical exploration research after 1998, the mechanism, significance, function and development of reading therapy was introduced thoroughly by Chinese scholars, and the idea of developing reading therapy service in libraries, especially in the university libraries and hospital libraries was discussed according to the actual situation in China, and the psychological intervention of reading therapy was preliminarily explored [6].

\section{1) Theory Research}

The new thought of expanding library services and library science research with books was put forward by Shen Guchao in 1998[7], and the principle of reading therapy was explained with the traditional Chinese medicine phase of emotion inter-resistance in the same year by Wang Bo, the history of bibliotherapy in China from ancient to modern was summarized in Bibliotherapy in China [8], which can be seen as the beginning of localization. The idea of carrying out reading therapy in the university libraries was put forward by Teacher Huang Juping in 1999 for the first time [9]. Since then, the study of reading therapy in universities for university students has become the most important part of reading therapy research.

\section{2) Practice Research}

Zhao Fengfeng used the concept of "reading therapy" and made an investigation study on reading therapy for the first time in 1999, and put forward the recommendation of "medical library should become the pioneer of reading therapy" and concluded the diseases and the required type of book [10]. The contrast experiments before and after reading therapy to 80 medical undergraduates of grade 2000 was carried out by TaiShan Medical College Library GongMeiLing for the first time in 2000, later this group of students were randomly divided into reading therapy class and comparative classes to make a comparative analysis research with the symptom self-assessment scale (SCL-90)to proof that reading therapy was significant in dealing with the common psychological problems of university students [11]. 


\section{System Deepening Stage}

Theoretical research deepened into system with the rapid development of reading therapy after 2004, empirical research applications were broader and achievements were remarkable, at the same time reading therapy also has been opened for medical profession and prisons profession.

\section{1) Theory Research}

The concept of reading prevention was used by Ju Mitai etc. for the first time and library reading self-service was brought into psychological barrier intervention model system of university students, which marked the official start of the psychological researchers involving in reading therapy research [12]. Teacher Wang Bo researched the type, concept, principle and bibliography of reading therapy in depth, and mined the long history of reading therapy in China from 2003 to 2006. His book Reading Therapy published in 2007, which promoted the development of reading therapy theory system greatly.

\section{2) Practice Research}

Gong MeiLing etc. did research into reading therapy practice constantly and has achieved gratifying achievement based on TaiShan Medical College: She started the bog of Book Therapy Hut on Sina.com in 2006, which expanded a new areas of network reading therapy service; guided students to create University Students Reading Therapy Study Association in the school in December 2007; established the reading therapy research base in 2008 [13]; Dr. Wan Yu put forward the reading therapy mode of "game + read+ feedback" based on the reading therapy practice in Diaoyutai Elementary School in Nanjing and helped some shy children build self-confidence and achieved a big success, which had innovation and sample value[14];Chen Shumei, explorer of reading therapy in library field in Taiwan, focused on the role of reading therapy in psychological reconstruction after natural disasters earlier. Activity of "Sending Children's Emotional Healing Books to Sichuan" was called for by her at once when the Wenchuan earthquake happened, and the book Bibliography of Children's Emotional Healing books published in December 2009[15]. Reading therapy service in Hebei United University (Jianshe Road Campus) Library (the original North China Coal Medical College Library) had a rapid development promoted by Chief Huang Xiaoli actively: Participated in the writing of the book Theory and Practice of Reading Therapy in 2010;Reading therapy work team with different disciplines background and different levels was set up in the same year, and reading therapy practice was carried out in depth in team collaboration mode in the school. Built domestic first reading therapy service web site, created publication Reading Therapy Work Newsletter, compiled bibliography of reading therapy, which created the pioneer of carrying out the work of reading therapy in team collaboration mode in China[16,17].

The value and potential of reading therapy also attracted the attention of medical profession and prisons profession. Chen Fei,etc. divided 120 cases of patients with schizophrenia into groups to control study, it was concluded that reading therapy was helpful for patients recovering self-knowledge, shortening the length of hospital stay, improving treatment adherence and reducing the recurrence rate [18]; Fan Wentian, etc. designed reading therapy intervention treatment on 103 cases of patients with depression, and summarized a variety of influenced factors of reading therapy to depression [19]. Reading therapy was also applied to reduce anxiety and depression in cancer patients, patients with gynecological surgery or other kinds of surgery. Qiu Hongzhong etc. confirmed that the reading therapy can alleviate inmates' anxiety effectively with experimental study [20].

\section{CONCLUSIONS AND DISCUSSION}

\section{A. Expand Reading Therapy Methods and Application Fields}

Reading therapy research in China presented diversified trend, but more was given priority to theoretical discussion, the proportion of empirical research was relatively small, and the narrative form of prose and case in empirical study was highly templated even homogeneous. Indications and applicable objects of reading therapy was many, there were a lot of researches to do for each indication and each type of applicable objects. Therefore, there was still a broad space for Reading therapy research in China. The ways, means, and application fields of reading therapy need to be expanded further.

\section{B. Carry Out The Multidisciplinary Joint Collaborative Research}

As a cross subject, reading therapy is attracting library science, medicine, psychology, pedagogy, sociology and other disciplines scholar's attention and participation, but reading therapy research in China is done in individual labor mostly, and though the researchers are many, backbone are less. Reading therapy research in China should overcome the low efficiency circulation within the narrow subject, carry out the multidisciplinary joint collaborative research, break through the original independent research ideas and methods, and then achieve systematic study comprehensively.

\section{Study Side Effects of Reading Therapy and Its Control in Depth}

Reading therapy is a kind of complex psychological therapy, a little knowledge about reading therapy and the patient may cause side effects. The case of patients with depression becoming worse in the process of treatment has noticed by Domestic scholars Gong Meiling [21].The aspects of study on side effects and its effective control, general treatment bibliography of reading therapy are less, and some achievements have yet to be tested.

\section{Carry Out Network Reading Therapy Research and Services}

The Internet has become the largest library around the world, western research experience can be got from a lot of information resources on the Internet, and the latest research results can be understood by dynamic database. In service practice, we can develop specialized website, recommend reading therapy bibliography, provide download links of relevant books, solve the consultant's psychological perplexity, carry out Internet counseling services, and answer for them through chat rooms, E-mail and online interactive features.

In a word, reading therapy, as a kind of both old and young psychotherapy, integrates prevention, treatment and healthy psychological development. Its application and promotion will be helpful for people to prevent mental illness, solve psychological problems and improve the 
psychological quality, and has a certain practical significance and far-reaching historical significance to the construction of a harmonious society, which should be the common concern of the whole society.

\section{REFERENCES}

[1] Wang Bo,Fu Xin.The Princle of Reading Therapy[J].Library.2003,(3):1.

[2] Xia Zhenyi.Chinese Medical Encyclopedia o:Psychiatry [M].ShanHai: Science and Technology Press.1982:135.

[3] Chen Xinchun.Literature Therapy — One of The Contents of The reader Service Work [J].Library Journal,1991 (2) :22.

[4] Yang Bing , Qiu Yousheng . Reading Therapy in American[J]. Library Tribune,1995, (4):75-76.

[5] Ling Shan, Hua Weina. Introduction of Reading Therapy [J]. Journal of Clinical Psychiatry, 1995, 5 (1) : 42-43.

[6] Shi Quansheng. Review of Reading Therapy Research For Twenty Years in China [J].Chinese Journal of Practical Nursing,2009,25 (7) : 19-21.

[7] Shen Guchao. Bibliotherapy :A New Field of Library Services and Library Science Research in China [J]. Library and Information Service, 1998, (4) : 12-15.

[8] Wang Bo. Biblioyherapy in China [J]. Chinese Library Journal, 1998(2):79-86.

[9] Huang Juping. Advice on Reading Therapy in University Library [J]. Jiangsu Journal of Library, 1999, (6) : 52-53.

[10] Zhao Fengfeng. Investigation and Recommendation to Reading Therapy [J]. Chinese Library Journal， 2000(1):38-39.

[11] Gong Meiling, CongZhong,Wang Lianyun,etc. Effect Evaluation of Reading Therapy to Solve Psychological Problems of College Students [J] . Chinese Journal of Behavioral Medical Science.2002,11(5):578.

[12] Ju Mitai, Zhang Jianxin, Yun Weiheng,etc. The Theoretical Set-up of the Intervention Mode for Undergraduates Suffering from Mental Disorder [J]. Psychological Science, 2005(6):1463-1467.

[13] Wang Xueyun. Gong Meiling and Her Team's Study and Practice on Reading Therapy [J]. Library Tribune, 2012, 32 (1) : 137-140,64.

[14] Wan Yu. Exploratory Practice of Reading Therapy in the Primary Stage - Nanjing DiaoYutai Elementary School Applications [J]. Library Journal, 2010 (10):37-41, 15.

[15] Wang Bo. The Latest Progress Reading Therapy Theory and Practice in China [J]. Library Journal, 2010(10):25-32.

[16] Wang Jingwen, Huang Xiaoli.Research on College Students' Reading Therapy Service Platform Resources Construction Based on Campus Network [J]Journal of Library Science, 2012,(11):35-36,46.

[17] Huang Xiaoli,Wang Jinwen. Consideration about the fundamental questions about reading therapy bibliographic compilation [J]. Journal of Academic Library and Information Science.2012,30(3) : 63-64,70.

[18] Chen Fei, Yang Yongjie,etc.. Effect of Bibilotherapy on Insight Recovering, Drug Therapy Compliance and Recurrence in Remitted Schizophrenia Patients [J]. Chinese Journal of Behavioral Medicine and Brain Science.2009(9):808-810.

[19] Fan Wentian,Wang Yang,Meng Xianfen, et al. A multiple factors analysis on efficacy of bibliotherapy for patients with depression in rehabilitation. [J]. Journal of Psychiatry, 2008(3):172-175.

[20] Qiu Hongzhong, Lu Danfeng,Wang Yuanrui,et al. Prospectne Study on Reading Therap to Mental Health Prisoners.[J].China Journal of Health Psychology, 2010(10):1197-1199.

[21] Gong Meiling. Practical Exploration of Reading Therapy in University [J]. Library Journal, 2010(10):33-36. 